\title{
Specialized multi-disciplinary heart failure clinics in Ontario, Canada: an environmental scan
}

\author{
Harindra C Wijeysundera ${ }^{1,2,3^{*}}$, Gina Trubiani ${ }^{2}$, Lusine Abrahamyan ${ }^{2}$, Nicholas Mitsakakis ${ }^{2}$, William Witteman², \\ Mike Paulden ${ }^{2}$, Gabrielle van der Velde ${ }^{2,4}$, Kori Kingsbury ${ }^{5}$ and Murray Krahn ${ }^{2,3,6,7}$
}

\begin{abstract}
Background: Multi-disciplinary heart failure (HF) clinics have been shown to improve outcomes for HF patients in randomized clinical trials. However, it is unclear how widely available specialized HF clinics are in Ontario. Also, the service models of current clinics have not been described. It is therefore uncertain whether the efficacy of HF clinics in trials is generalizable to the HF clinics currently operating in the province.
\end{abstract}

Methods: As part of a comprehensive evaluation of HF clinics in Ontario, we performed an environmental scan to identify all HF clinics operating in 2010. A semi-structured interview was conducted to understand the scope of practice. The intensity and complexity of care offered were quantified through the use of a validated instrument, and clinics were categorized as high, medium or low intensity clinics.

Results: We identified 34 clinics with $143 \mathrm{HF}$ physicians. We found substantial regional disparity in access to care across the province. The majority of HF physicians were cardiologists (81\%), with $81 \%$ of the clinics physically based in hospitals, of which $26 \%$ were academic centers. There was a substantial range in the complexity of services offered, most notably in the intensity of education and medication management services offered. All the clinics focused on ambulatory care, with only one having an in-patient focus. None of the HF clinics had a home-based component to care.

Conclusions: Multiple HF clinics are currently operating in Ontario with a wide spectrum of care models. Further work is necessary to understand which components lead to improved patient outcomes.

\section{Background}

Heart failure (HF) is a complex, progressive syndrome characterized by abnormal heart function resulting in poor exercise tolerance, recurrent hospitalizations, and reductions in both quality of life, and survival [1]. Although tremendous progress has been made in pharmacologic and device therapy, HF patients continue to have a poor prognosis, with an annual mortality ranging from $5 \%$ to $50 \%$ [1]. The incidence of HF is projected to increase, with estimates suggesting a three-fold increase in HF hospitalizations over the next decade [2]. Alternative targeted health care delivery models have therefore been

\footnotetext{
*Correspondence: wijeysundera@gmail.com

'Division of Cardiology, Schulich Heart Centre, Sunnybrook Health Sciences Centre, Ontario, Canada

${ }^{2}$ Toronto Health Economics and Technology Assessment (THETA)

Collaborative, Ontario, Canada

Full list of author information is available at the end of the article
}

of particular interest in HF, as a means of improving both quality of life and survival [3].

Disease management through multi-disciplinary community care clinics has been shown to improve patient outcomes in different health conditions, including diabetes, chronic kidney disease, and cancer $[4,5]$. The potential benefits of a multi-disciplinary strategy in HF include improved utilization and adherence with evidence-based medications. This model of care may also address the complex interplay between medical, psychosocial, and behavioural factors facing these patients and their caregivers [3]. Multiple previous randomized studies and metaanalyses have evaluated the efficacy of such clinics with some suggesting a reduction in mortality in excess of $20 \%$ $[1,3,6]$. However, interpreting this literature is challenging because of substantial heterogeneity in the composition of the HF clinics, the interventions they offer, and the population studied [3,7].

\section{() BioMed Central}

(c) 2012 Wijeysundera et al.; licensee BioMed Central Ltd. This is an Open Access article distributed under the terms of the Creative Commons Attribution License (http://creativecommons.org/licenses/by/2.0), which permits unrestricted use, distribution, and reproduction in any medium, provided the original work is properly cited. 
Currently, specialized HF clinics do not receive specific funding from the Ontario Ministry of Health and Long Term Care (MOHLTC), the third party payer for government insured health services in the province. It is not known how widely available specialized HF clinics are in Ontario, nor has their composition, or the services they offer, been described. Therefore, it is unclear if the efficacy of HF clinics in randomized trials is generalizable to the HF clinics currently in place in Ontario. Our objective was to address these important gaps in knowledge, through a comprehensive field evaluation, whereby real world practice for HF patients in Ontario was assessed in 2010. Specially, we aimed to understand the current availability of specialized HF clinics in the province, and the intensity and complexity of services offered.

\section{Methods}

Canada is divided into 13 distinct territories or provinces, with Ontario being the most populous. Based on the most recent census, 12.2 million of Canada's 31.6 million people lived in Ontario. The Ontario population is concentrated around major urban areas, with only $15 \%$ living in rural settings, defined as a population less than 1000 persons and less than 400 persons per $\mathrm{km}^{2}$.

There is universal access to medical care in Canada without user-fees or out-of-pocket payments. Health care funding is determined at the provincial level. In 2006, the Ontario Ministry of Health and Long-Term Care transferred the responsibility for planning, integrating and funding of health services within the province to 14 regional Local Health Integration Networks (LHIN).

\section{Identification of Heart Failure Clinics}

For the purpose of this project, a specialized HF clinic was defined as a clinic that consists at a minimum of a physician and a nurse, one of whom has specialized training/interest in HF. This definition is consistent with that used in recent systematic reviews of HF clinics [8].

We utilized three approaches to identify clinics. First, all hospitals listed on the MOHLTC site (www.health. gov.on.ca) were contacted. Notices were posted in the Cardiac Care Network (CCN) webpage. Finally, we used snow-ball sampling, an approach often used in qualitative or mixed methods research studies, to evaluate 'hidden populations' [9].

A hidden population is one in which a sample frame (i.e. a list of all the members of the population) cannot be constructed, thereby preventing probability sampling [9]. An alternative that does not require a sampling frame is snow-ball sampling, whereby new members are selected from the social network of existing members of the sample [9].

In this method, a number of seeds are first selected [9]. These seeds are members of the hidden population that have been identified. The seeds are interviewed and form stage 0 of the sampling process. The seeds identify other members of the population, who are in turn approached in the next generation of sampling (stage 1). This process is continued until the desired sample size is reached. This method has been successfully utilized in a myriad of cardiac studies [10-12].

In our study, the initial seeds were the Ontario members of the Canadian Heart Failure Network (CHFN) and other sites identified by the expert panel (Table 1). Established in 1999, the CHFN is a network of academic and community based clinics that provide specialized care to HF patients (www.cfna.ca). Importantly, the network did not include all HF clinics in the province, thereby necessitating further sampling.

The physician or nursing lead at each clinic was approached and a semi-structured interview conducted to establish the scope of the practice. The lead was asked to identify any other HF clinics, which may serve patients in the vicinity $\left(1^{\text {st }}\right.$ sampling stage). We continued to accrue new sampling stages until no new clinics were identified, at which point the sample was saturated.

\section{Regional differences in access to HF Clinics}

The boundaries of each LHIN were used to assess any geographic inequalities in access to HF clinics. We first determined the population size overall and of persons greater than the age of 65 years in each LHIN. The number of prevalent HF cases in each LHIN is not known. To approximate the burden of HF per LHIN, we used previously published data on the number of hospital discharges per LHIN with a most responsible diagnosis of HF in the

\section{Table 1 Seed heart failure clinics}

\begin{tabular}{l} 
Clinic name and location \\
\hline 1. Cornwall: Cornwall Community Hospital \\
2. Hamilton: Heart Function Clinic - Hamilton Health Sciences \\
3. Kingston: Hotel Dieu Hospital \\
4. Kitchener: St. Mary's Hospital \\
5. London: London Health Sciences Centre \\
6. Oakville: Oakville-Trafalgar Memorial Hospital \\
7. Orillia: Orillia Soldiers' Memorial Hospital \\
8. Ottawa: University of Ottawa Heart Institute \\
9. Owen Sound: Grey Bruce Health Services \\
10. Picton: Prince Edward Family Health Team Heart Failure Clinic \\
11. Toronto: University Health Network (UHN) (1) \\
12. Toronto: University Health Network (UHN) (2) \\
13. Toronto: Mt Sinai Heart Function Clinic \\
14. Toronto: St Michael's Hospital Heart Function Clinic \\
15. Toronto: Sunnybrook Hospital Heart Function Clinic
\end{tabular}


fiscal years 1997-2001 [13]. We then determined the annual rate of HF hospital discharges per HF clinic in each LHIN as another estimate of the regional distribution of access to care.

\section{Semi-structured Interview}

The semi-structured interview ascertained information broadly on the characteristics of the clinics themselves and the program service model. We used the HF Disease Management Scoring Instrument (HF-DMSI), a validated questionnaire developed by Riegel and colleagues to measure the intensity and complexity of each clinic's program service model across 10 categories [14]. Details on the categories and the respective scoring algorithm are found in Table 2 [14]. Two researchers (GT, WW) independently scored each clinic based on the interview transcripts.

Briefly, the HF-DMSI focused on the composition of the HF team (single practitioner vs. multi-disciplinary team) and the content of the HF intervention such as education (scored from 0 to 4 , with 4 as the more comprehensive education program), and medication management (scored from 0 to 3 ). The environment of the HF clinics was categorized as those that only focused on inpatients with HF (score of 1) versus those that focused only on outpatients seen in clinic (score of 2), those that were home-based with the intervention taking place in the patients' residence (score of 3), with clinics that had components in more than one setting receiving the highest score of 4 . Peer support, remote monitoring, and the duration and complexity of contact were also measured. The instrument was designed to provide a separate score for each category. The HF-DMSI has content validity and an excellent inter-rater reliability with a intra-class correlation coefficient of 0.918 [14].

Because the HF-DMSI does not provide an overall summary score, and could not be used to rank clinics, we performed a concept mapping exercise, using an HF expert panel. The concept mapping exercise consisted of two parts $[15,16]$. In part 1 , we determined the relative importance of each of the 10 categories of the HF-DMSI, based on consensus of the expert panel. In the second part, each of the clinics identified were categorized by the expert panel into three intensity groups, based on their scores on the HF-DMSI, influenced by the implicit weighting system revealed in part 1 . Further description of this process is found in Additional file 1 Appendix A.

\section{Institutional review board}

The ethics review board of the University of Toronto approved this protocol. When required by local institutional regulations, separate institutional review board approval was acquired for each participating clinic. Consent for the use of the structure survey results was obtained from the physician lead for each identified HF clinic.

\section{Results}

\section{HF clinic identification}

Between May 2010, and August 2010, we identified a total of 34 clinics through our sampling method, as seen in Figure 1. From the initial 15 seed clinics identified through the CHFN, three generations of snow-ball sampling took place, at which point the sample was saturated. Five clinics were identified through the $\mathrm{CCN}$ and one HF clinic through contacting individual hospitals. Of these clinics, 30 agreed to participate in the semistructured survey.

\section{Regional distribution of HF clinics}

The initial seed clinics were located in 9 of the Ontario 14 LHIN's. We were able to identify HF clinics in all the remaining LHINs except for the Central West and Erie St Clair LHINs. There was substantial regional variation in access to HF clinics. As apparent from Figure 2 and Table 3, the identified HF clinics were concentrated in the south and central regions of the province. Each HF clinic served an average population of 353,800 with an over 65-year-old population of 45,200. However, there was a substantial range in the population served in the LHINs with identified HF clinics, from 179,200 per clinic in the Toronto Central LHIN, to 761,400 in the central LHIN.

In order to estimate the burden of HF across Ontario, we used data which showed over the 5 years from 1998 to $2002,42,367$ patients were discharged with a diagnosis of HF. As seen in Table 3, given the 34 clinics, on average each HF clinic would be able to serve $200 \mathrm{HF}$ discharges per year. However, there was substantial regional variability, with greater than a 7 fold difference between LHINs with HF clinics. For example in Waterloo, there was a HF clinic for $84 \mathrm{HF}$ discharges, compared to one HF clinic per $626 \mathrm{HF}$ discharges in the North East LHIN.

\section{Clinic characteristics}

Identified HF clinics had a mean of 138 new consults (median 78; interquartile range 25-128) and 1020 visits per year (median 675; interquartile range 200-1479). However, there was substantial variation in their service volume, as evidence in Figure 3, with two high volume clinics which were outliers (clinic \#17 and \#25). Clinic \#25 had 4900 annual visits, with 1400 new patients per year. Clinic \#17 had 4200 annual visits, but only 350 new patients annually. In contrast to the other HF clinics, the majority of patients seen at clinic 4 were new (represented by the red bar), with only a limited number of follow-up visits (represented by the blue bar). 
Table 2 Heart failure disease management scoring instrument (HF-DMSI)

\begin{tabular}{|c|c|}
\hline Intervention category & Points to be assigned \\
\hline \multirow[t]{4}{*}{ Recipient } & $1=$ Provider alone \\
\hline & $2=$ Patient alone \\
\hline & $3=$ Patient with some inclusion of caregiver \\
\hline & $4=$ Patient with a caregiver who is central to the intervention \\
\hline \multicolumn{2}{|l|}{ Intervention content } \\
\hline \multirow{5}{*}{$\begin{array}{l}\text { Education and counselling aimed at } \\
\text { supporting self-care }\end{array}$} & $0=$ No mention of education \\
\hline & $1=$ Focus solely on importance of treatment adherence \\
\hline & $2=$ Focus on treatment adherence including some creative methods of improving adherence \\
\hline & $\begin{array}{l}3=\text { Focus on surveillance but no mention of actions to be taken in response to symptoms (eg, no } \\
\text { flexible diuretic management) }\end{array}$ \\
\hline & $\begin{array}{l}4=\text { Emphasis on surveillance, management, and evaluation of symptoms in addition to treatment } \\
\text { adherence }\end{array}$ \\
\hline \multirow[t]{4}{*}{ Medication management } & $0=$ No mention of medication regimen \\
\hline & $\begin{array}{l}1=\text { Some mention of medications (eg, importance of medication compliance) but not an active part of } \\
\text { the intervention. No attempt to intervene with provider to get patients on an evidence-based } \\
\text { medication regimen }\end{array}$ \\
\hline & $\begin{array}{l}2=\text { Evidence-based medication regimen advocated but no follow-up with patient or provider to } \\
\text { monitor the suggestion }\end{array}$ \\
\hline & $\begin{array}{l}3=\text { Medication regimen monitored, attempt made to get the patient on evidence-based medications, } \\
\text { with follow-up monitoring done with patient or provider }\end{array}$ \\
\hline \multirow[t]{3}{*}{ Social support Peer support } & $0=$ No mention of a peer support intervention \\
\hline & $1=$ Peer support mentioned but not integral to intervention \\
\hline & $2=$ Peer support integral component of intervention \\
\hline \multirow{3}{*}{$\begin{array}{l}\text { Surveillance by provider:Remote } \\
\text { monitoring }\end{array}$} & $0=$ No use of remote monitoring or telehealth \\
\hline & $\begin{array}{l}1=\text { Remote monitoring is used in conjunction with other interventions that form the main intervention } \\
\text { used }\end{array}$ \\
\hline & $2=$ Telehealth is essential component of intervention \\
\hline \multirow[t]{3}{*}{ Delivery personnel } & $1=$ Single generalist provider (eg, physician, nurse, pharmacist) \\
\hline & $2=$ Single HF expert provider (eg, physician, nurse, pharmacist) \\
\hline & $3=$ Multidisciplinary intervention \\
\hline \multirow[t]{4}{*}{ Method of communication } & $1=$ Mechanized via internet or telephone \\
\hline & 2 = Person-to-person by telephone \\
\hline & $3=$ Face-to-face, individual, or in a group \\
\hline & $\begin{array}{l}4=\text { Combined: Face-to-face at least once alone or in a group with individual telephone calls in between } \\
\text { meetings }\end{array}$ \\
\hline \multicolumn{2}{|l|}{ Intensity and complexity } \\
\hline \multirow[t]{4}{*}{ Duration } & $1=\leq 1 \mathrm{mo}$ \\
\hline & $2=\leq 3 \mathrm{mo}$ \\
\hline & $3=\leq 6 \mathrm{mo}$ \\
\hline & $4=>6 \mathrm{mo}$ \\
\hline \multirow[t]{3}{*}{ Complexity } & $1=$ Low: single contact with little or no follow-up \\
\hline & $2=$ Moderate: $>1$ but $<4$ and/or infrequent contact or contacts of short duration \\
\hline & $3=$ High: multiple contacts of significant duration \\
\hline \multirow[t]{4}{*}{ Environment } & 1 = Hospital: Inpatient only \\
\hline & $2=$ Clinic/outpatient setting \\
\hline & $3=$ Home-based \\
\hline & $4=$ Combination of settings \\
\hline
\end{tabular}




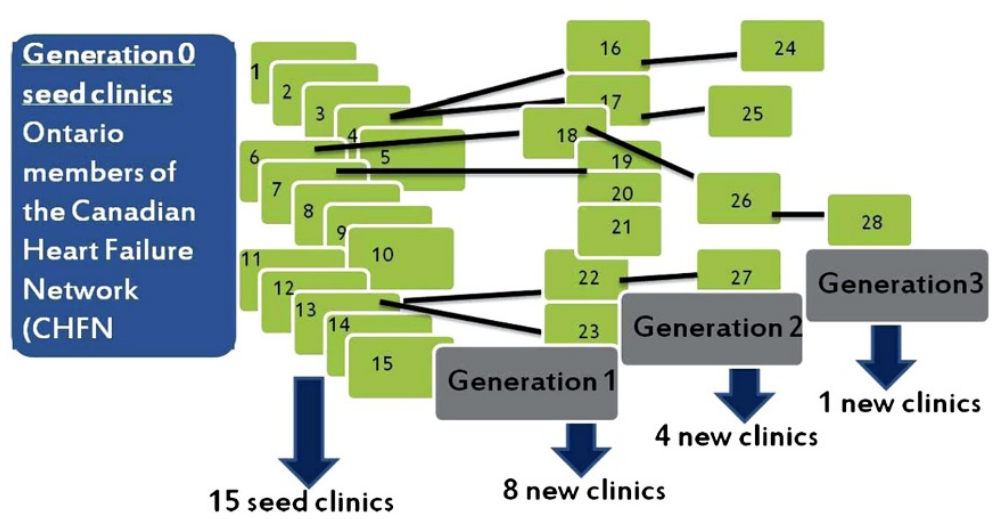

Figure 1 Process by which 28 clinics were identified by snowball sampling. Based on interview responses from the initial 15 seed clinics, full saturation was reached in 3 generations.

The majority (80.6\%) of clinics were physically based in hospitals with $25.8 \%$ being part of an academic institution. In total, $143 \mathrm{HF}$ clinic physicians worked at the 30 identified clinics. The majority of clinics were run by cardiologists.

\section{Access to allied health professionals}

The clinics had on average limited access to in-clinic allied health professionals, as seen in Table 4. Under half had access to dieticians or pharmacists, with only $6.5 \%$ and $16.1 \%$ with in-clinic access to physiotherapists or

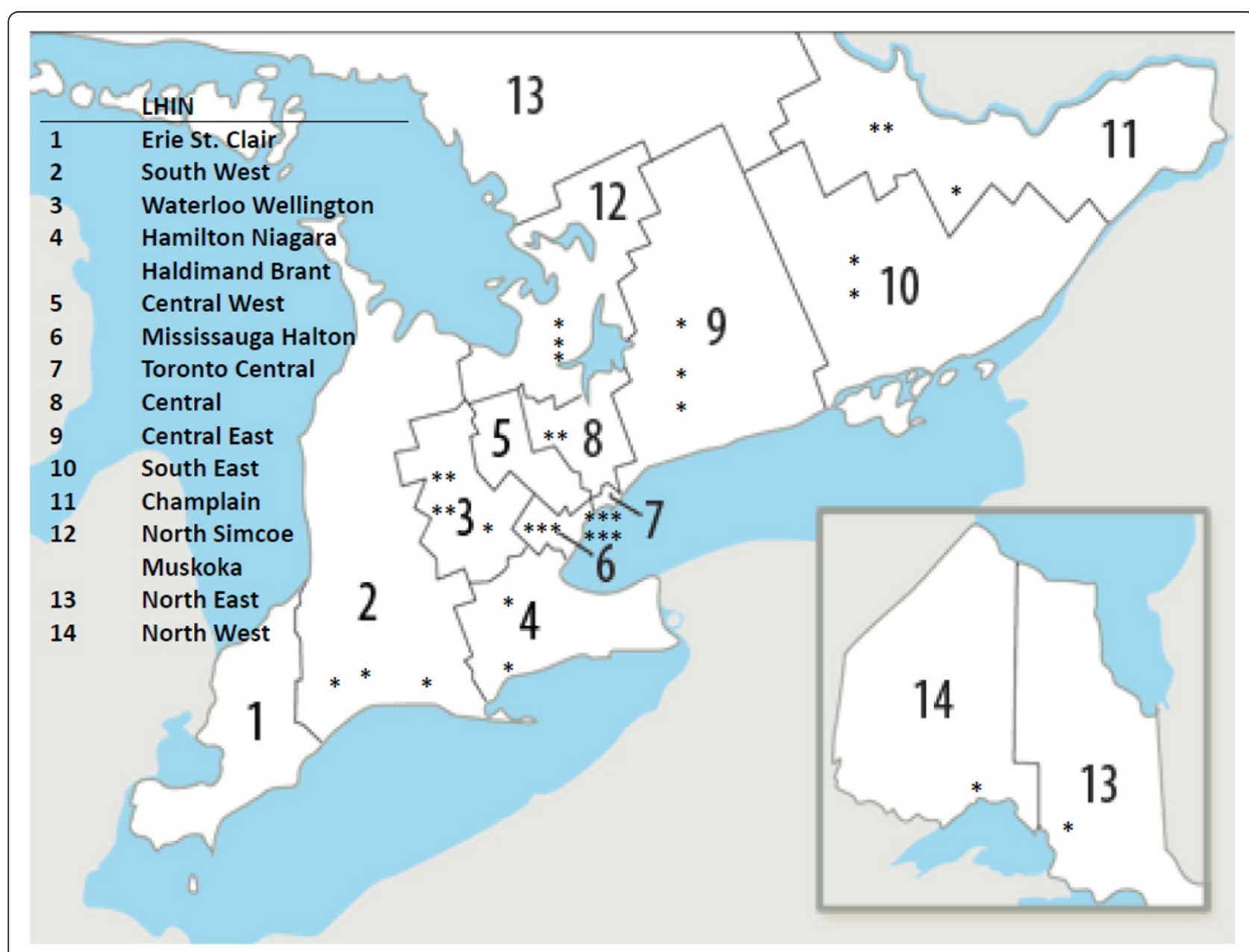

Figure 2 Regional Local Health Integration Networks (LHIN) in Ontario depicting regional distribution of identified heart failure clinics. 
Table 3 Geographic distribution of clinics

\begin{tabular}{|c|c|c|c|c|c|c|}
\hline LHIN & $\begin{array}{l}\text { \# HF } \\
\text { Clinics }\end{array}$ & $\begin{array}{c}\text { Total } \\
\text { Population }\end{array}$ & $\begin{array}{l}\text { population per } \\
\text { HF Clinic }\end{array}$ & $\begin{array}{c}\text { age } 65 \text { years and over } \\
\text { in LHIN }\end{array}$ & $\begin{array}{c}>65 \text { years population per } \\
\text { HF clinic }\end{array}$ & $\begin{array}{c}\text { annual HF discharge per } \\
\text { HF clinic }\end{array}$ \\
\hline Erie St. Clair & 0 & 623,300 & NA & 85,000 & NA & NA \\
\hline South West & 3 & 890,100 & 296,700 & 125,800 & 41,900 & 247 \\
\hline $\mathrm{HNHB}$ & 2 & $1,298,300$ & 649,100 & 192,400 & 96,200 & 591 \\
\hline Waterloo Wellington & 5 & 679,700 & 135,900 & 76,000 & 15,200 & 84 \\
\hline Mississauga Halton & 3 & $1,002,300$ & 334,100 & 103,400 & 34,500 & 155 \\
\hline Central West & 0 & 735,200 & NA & 65,900 & NA & NA \\
\hline Central & 2 & $1,522,800$ & 761,400 & 183,100 & 91,600 & 395 \\
\hline Central East & 3 & $1,419,800$ & 473,300 & 184,600 & 61,500 & 305 \\
\hline Toronto Central & 6 & $1,075,100$ & 179,200 & 131,800 & 22,000 & 118 \\
\hline North Simcoe Muskoka & 3 & 417,000 & 139,000 & 59,900 & 20,000 & 108 \\
\hline South East & 2 & 457,200 & 228,600 & 74,700 & 37,400 & 217 \\
\hline Champlain & 3 & $1,131,400$ & 377,100 & 137,600 & 45,900 & 247 \\
\hline North East & 1 & 545,000 & 545,000 & 84,900 & 84,900 & 626 \\
\hline North West & 1 & 231,900 & 231,900 & 31,400 & 31,400 & 218 \\
\hline Total & 34 & $12,028,900$ & 353,800 & $1,536,500$ & 45,200 & 200 \\
\hline
\end{tabular}

LHIN: Local Health Integration Network; HF: Heart Failure; HNHB: Hamilton Niagara Haldimand Brant; NA: not applicable.

counsellors. $87.1 \%$ of HF clinics had a formal affiliation with a cardiac rehabilitation program and $64.5 \%$ where actively involved with chronic disease management of another condition, such as diabetes mellitus.

\section{Intensity and complexity}

The ranges of HF clinic scores on the HF-DMSI are shown on Figure 4. There was little variation between the clinics for some elements of the instrument, such as intervention duration (all scored 4; greater than 6 months). The majority of HF clinics had a formal medication management protocol, where medications were monitored and an attempt was made to increase utilization of evidence-based medications. There was substantial range in the intensity of education and counselling aimed at supporting self-care. Although all clinics had some form of education program, these ranged from programs that focused only on adherence to more comprehensive programs that emphasized surveillance, management and evaluation of symptoms in addition to treatment adherence. The majority of clinics did not use remote monitoring at the clinic, although half did contact patients by telephone in between face-to-face evaluations. A formal peer support component was identified in only one HF clinic. Somewhat surprisingly, although the delivery personnel at the clinic were multidisciplinary in approximately $50 \%$ of clinics, some had only either a single generalist or HF expert provider. As far as environment, all of the clinics were ambulatory based, with one that was predominantly focussed on inpatients. None were exclusively home-based or had a home-based component.

\section{Concept mapping}

Based on our concept mapping exercise, the expert panel categorized the 30 identified clinics into three strata of intensity; 8 clinics were assigned to the low intensity category, with 12 in the medium intensity category and 10 in the high intensity group. The mean scores on the HF-DMSI for these three strata are shown in Table 5. Although the high intensity clinics had higher mean scores in 9 of the 10 HF-DMSI categories, this was most pronounced in the education and counselling, medication management, delivery personnel and complexity categories. This suggests an implicit weighting of these categories by our expert panel as revealed by the concept mapping exercise. In contrast, remote monitoring and the presence of a structured peer-support program were believed to be of lesser importance.

\section{Discussion}

In this environmental scan of HF clinics in the province of Ontario, Canada, we were successfully able to identify 34 HF clinics. There was substantial inequity in access to care, with two LHINs having no identified HF clinics, and a wide range in the population served by each clinic. As anticipated, the clinics were varied in structure and the services offered. The greatest variation in terms of intensity and complexity was in terms of the education service offered. Remote monitoring and a home-base 

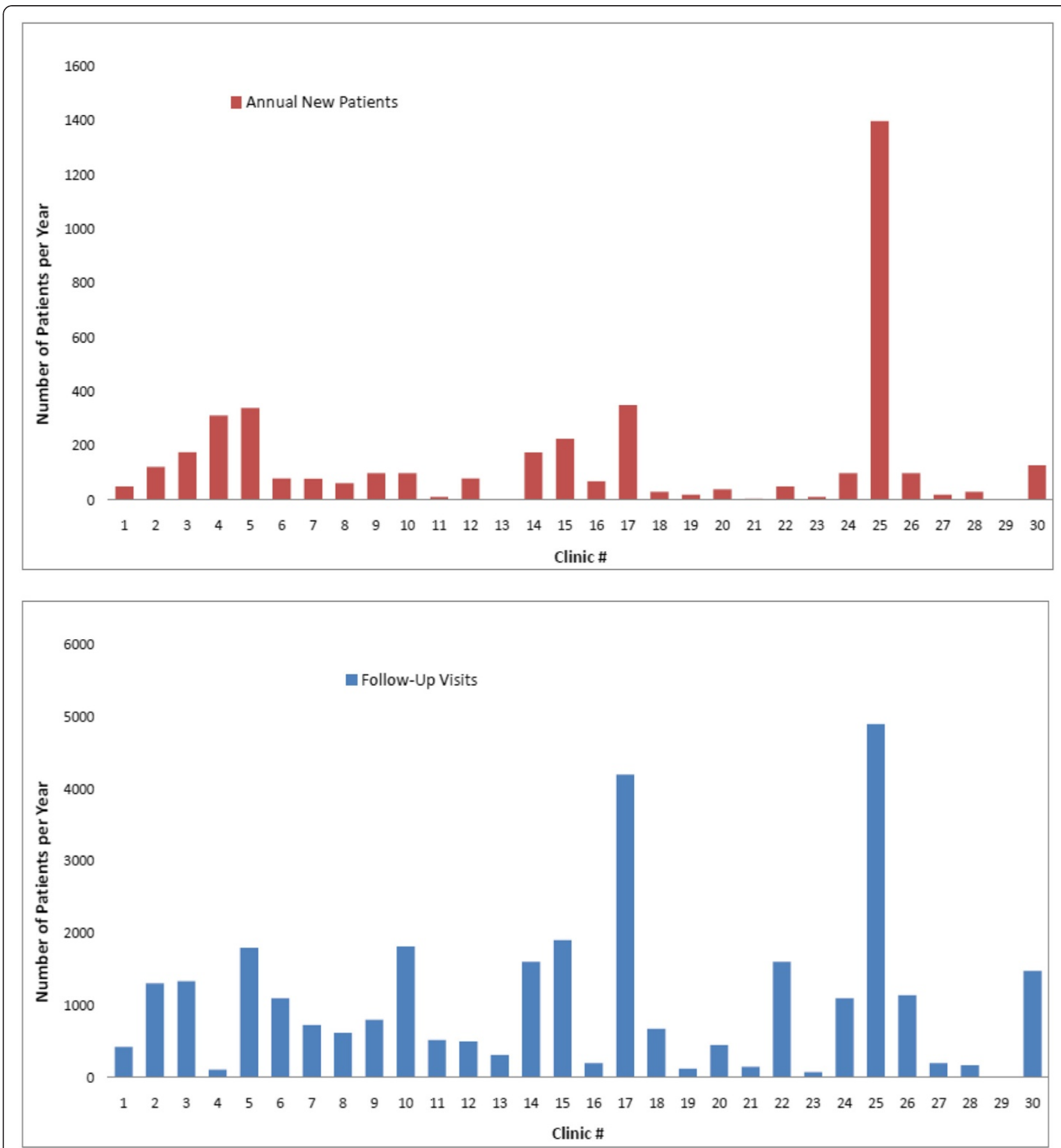

Figure 3 Annual Service Volume of the identified Heart Failure Clinics. The red bar indicates new patients per year, and the blue bar represents annual patient visits.

component to the HF clinic services were notably absent in most clinics.

Multi-disciplinary ambulatory complex disease management clinics are increasingly studied as the preferred modality of ambulatory care delivery for chronic diseases such as HF $[1,3,4,6,17]$. Advocates of such clinics highlight the many randomized clinical trials that show the efficacy of such clinics in reducing mortality and rehospitalisation [3,18-31]. Importantly, although these clinics are grouped together in systematic reviews and meta-analyses, there is heterogeneity in the models evaluated and services offered [7]. Prior to implementing these clinics in routine practice, it is critical to understand which components are central to the intervention. Several meta-analyses have 
Table 4 Characteristics of 30 identified clinics

\begin{tabular}{|c|c|}
\hline \multicolumn{2}{|l|}{ Parameter } \\
\hline PERSONEL & \\
\hline Mean number of Physicians & $4.7(1-8)^{*}$ \\
\hline$\%$ of clinics with cardiologist & 80.6 \\
\hline$\%$ of clinics with internists & 22.6 \\
\hline$\%$ of clinics with family physicians & 9.7 \\
\hline$\%$ of physicians with heart failure training & 80.6 \\
\hline Mean Number of Nurses & $2.0(1-6)^{*}$ \\
\hline \multicolumn{2}{|l|}{ LOCATION } \\
\hline$\%$ Academic & 25.8 \\
\hline$\%$ Community Based & 74.2 \\
\hline Mean Annual Total Visits & $1020(200-1479)^{*}$ \\
\hline Mean Annual Total New Patients & $139(25-128)^{*}$ \\
\hline$\%$ Access to Onsite Echocardiography & 80.6 \\
\hline$\%$ Access to Onsite Nuclear Cardiology Testing & 58.1 \\
\hline$\%$ Access to Onsite Angiography & 38.7 \\
\hline$\%$ Access to Onsite exercise Stress Testing & 77.4 \\
\hline Mean Exam Rooms & $3.3(1-4)^{*}$ \\
\hline \multicolumn{2}{|l|}{ ALLIED HEALTH PROFESSIONALS } \\
\hline$\%$ Access to Dietician (In Clinic) & 45.2 \\
\hline \% Access to Pharmacist (In Clinic) & 32.3 \\
\hline \% Access to Physiotherapy (In Clinic) & 6.5 \\
\hline$\%$ Access to Counselor (In Clinic) & 16.1 \\
\hline$\%$ Affiliated with Cardiac Rehabilitation & 87.1 \\
\hline$\%$ Involved in other Chronic Disease Management & 64.5 \\
\hline
\end{tabular}

attempted to address this research question using the published literature [3,18-22,27,28]. McAlister and colleagues evaluated 29 trials enrolling a total of 5,039 patients [3]. Because of substantial heterogeneity, they did not report an overall summary statistic [3]. They found that multi-disciplinary clinics improved mortality, while tele-monitoring improved re-hospitalization rates [3]. Holland and colleagues contrasted studies that incorporated home visits, or between visits telephone calls, to those that were solely hospital or clinic based [27]. In the 30 trials that were included in their analysis, they found that reductions in hospitalization were limited to studies that included either a home-based or telephone based component to the intervention.

Our study provides a number of insights for policy makers who are planning the implementation of such disease management systems in other regions. The proliferation of heart failure clinics in Ontario has occurred without specific guidance as to their structure, in part due to the absence of dedicated funding. This has resulted in considerable variation in important components such as education, and the notable absence of key features such as a home-based component or remote monitoring. Our findings are consistent with that seen by Driscoll and colleagues who found substantial variation in the care provided at HF management programs across Australia, raising concerns about the quality of care provided to these patients [32].

Understanding the association between heterogeneity in clinic model and outcomes such as mortality and rehospitalization is the logical next step in order to address if quality of care is compromised by this variation

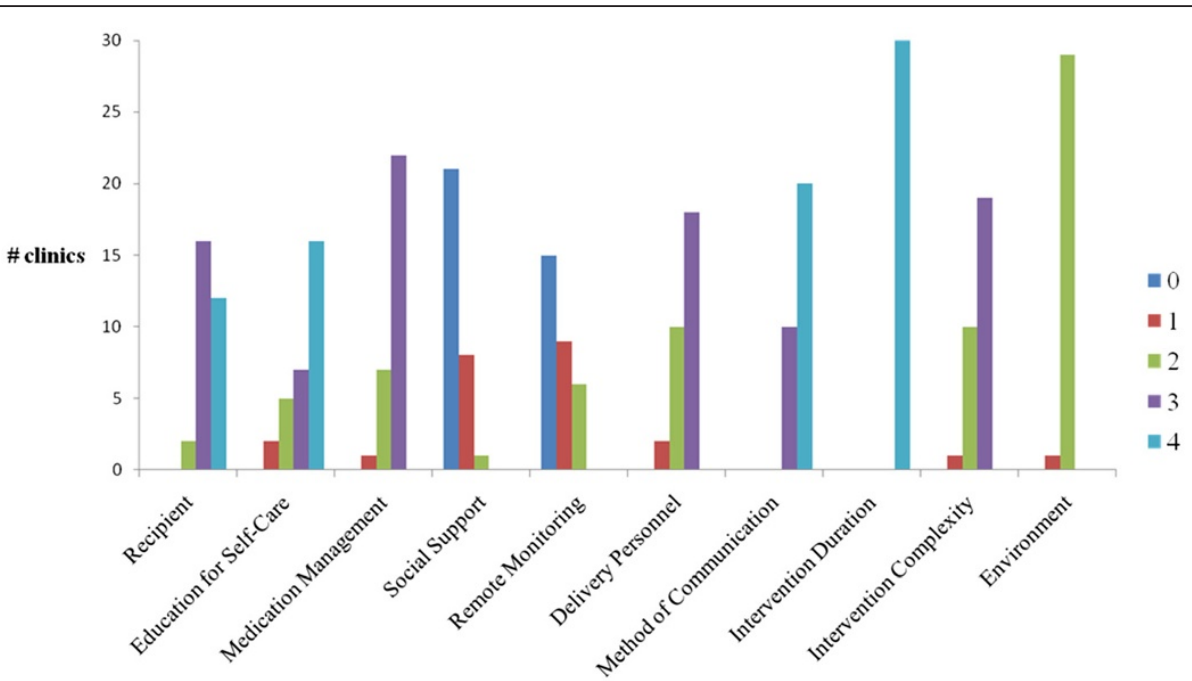

Figure 4 Distribution of scores on 10 categories of Heart Failure Disease Management Scoring Instrument (HF-DMSI). Please refer to Table 2 for specific definitions of individual scores. Higher scores indicate more comprehensive program within that category. 
Table 5 Clinic intensity and complexity

\begin{tabular}{|c|c|c|c|c|c|}
\hline \multirow[t]{2}{*}{ HF-DMSI category } & \multirow{2}{*}{$\begin{array}{c}\text { All } \\
\text { clinics } \\
(n=30)\end{array}$} & \multicolumn{3}{|c|}{ Clinic intensity types } & \multirow[b]{2}{*}{$\begin{array}{c}\mathrm{p}- \\
\text { value }\end{array}$} \\
\hline & & $\begin{array}{l}\text { High } \\
(n=10)\end{array}$ & $\begin{array}{c}\text { Medium } \\
(n=13)\end{array}$ & $\begin{array}{c}\text { Low } \\
(n=7)\end{array}$ & \\
\hline Recipient & $3.3 \pm 0.6$ & $3.7 \pm 0.5$ & $3.2 \pm 0.6$ & $3.0 \pm 0.6$ & .040 \\
\hline $\begin{array}{l}\text { Education and counselling aimed at } \\
\text { supporting self-care }\end{array}$ & $3.2 \pm 1.0$ & $3.9 \pm 0.3$ & $3.1 \pm 1.0$ & $2.6 \pm 1.1$ & .011 \\
\hline Medication management & $2.7 \pm 0.5$ & $3.0 \pm 0$ & $2.8 \pm 0.4$ & $2.1 \pm 0.7$ & .002 \\
\hline Peer support & $0.3 \pm 0.5$ & $0.6 \pm 0.7$ & $0.2 \pm 0.4$ & $0.3 \pm 0.5$ & .147 \\
\hline Remote monitoring & $0.7 \pm 0.8$ & $1.0 \pm 0.8$ & $0.8 \pm 0.8$ & $0.1 \pm 0.4$ & .079 \\
\hline Delivery personnel & $2.5 \pm 0.6$ & $3.0 \pm 0$ & $2.5 \pm 0.5$ & $2.0 \pm 0.8$ & .002 \\
\hline Method of communication & $3.6 \pm 0.5$ & $4.0 \pm 0$ & $3.5 \pm 0.5$ & $3.4 \pm 0.5$ & .018 \\
\hline Duration & $4.0 \pm 0$ & $4.0 \pm 0$ & $4.0 \pm 0$ & $4.0 \pm 0$ & - \\
\hline Complexity & $2.6 \pm 0.6$ & $3.0 \pm 0$ & $2.6 \pm 0.5$ & $2.0 \pm 0.6$ & $<.001$ \\
\hline Environment & $2.0 \pm 0.2$ & $2.0 \pm 0$ & $1.9 \pm 0.3$ & $2.0 \pm 0$ & .536 \\
\hline
\end{tabular}

HF-DMSI : Heart Failure Disease Management Scoring Instrument. Results are presented as means \pm standard deviations. Please refer to Table 2 for detail description of HF-DMSI categories and scoring.

in care models. In patients discharged after a $\mathrm{HF}$ hospitalization who were treated at HF clinics, we observed a 1-year mortality of $22.8 \%$ and a 1-year rehospitalisation rate for $\mathrm{HF}$ of $44.2 \%$. There was a striking 1.5 fold variation in mortality between clinics and a 2.5 fold variation in re-hospitalization rates. This highlights the need to identify which clinic-level components are predictive of improved outcomes, such that one can provide clinicians and policy-makers clear guidance when designing specialized HF clinics. These are foci of further research for our group.

Disease management through specialized HF clinics is recommended by guidelines for patients recently hospitalized with HF or at high risk for decomposition $[6,17]$. Currently, there is a paucity of data on what proportion of these patients are indeed seen at HF clinics. Although, this study was not designed to address this question, based on our estimates of annual HF discharges in the province and the annual number of new patients seen in HF clinics, it is likely that an only small proportion of appropriate patients are cared for at HF clinics. This is consistent with data from Australia, which suggests only $20 \%$ of eligible HF patients are seen at specialized HF clinics [32]. In addition, the catchment area served by each HF clinic (353,800 persons) in our study is larger than that seen in others surveys, such as one in Denmark ( 1 HF clinic per 115,000 persons) suggesting that there is less access in Ontario compared to other regions [32,33]. Moreover, our environmental scan suggests that there is substantial variation in access to HF clinics across the province. The absence of specific MOTHLC funding for the HF clinics may be a contributing factor. Elucidation of the underlying mechanisms for this disparity will be important for policy makers.
Our study must be interpreted in the context of several limitations. First, although we used a number of different methods to locate all HF clinics in the province, we cannot confirm that all clinics were in fact identified. We used an instrument to evaluate intensity and complexity; this did not cover all potential service components. Indeed, it does not include post-discharge planning, which has been identified by some studies as a critical component to reduce early rehospitalisation. Finally, although we have categorized clinics into intensity strata based on expert opinion, the relevance of such categories is dependent on their association with improved patient outcomes.

In summary, through our environmental scan, we found that despite the absence of specific governmental funding, there are at least 34 HF multidisciplinary clinics in operation in the province of Ontario. These clinics have a wide range of services offered. Further research on understanding which of these service components are associated with improved patient outcomes will aid policy makers and clinicians to determining the optimal care model for these complex patients.

\section{Additional file}

Additional file 1: Appendix A. Heart Failure Clinic Stratification using Concept Mapping.

\section{Competing interests}

None of the authors have any conflicts of interest to declare.

\section{Authors' contributions}

HCW Conception, design, acquisition, analysis and interpretation of data; drafting of manuscript; final approval of manuscript submitted. GT Design, acquisition and analysis of data; drafting of manuscript; final approval of manuscript submitted. LA Acquisition and analysis of data; revising of manuscript; final approval of manuscript submitted. NM Conception, design, 
analysis and interpretation of data; drafting of manuscript; final approval of manuscript submitted. WW Acquisition of data; revising of manuscript; final approval of manuscript submitted. MP Analysis, and interpretation of data; revising of manuscript; final approval of manuscript submitted. GrV Conception and design; revising of manuscript; final approval of manuscript submitted. KK Conception and design; revising of manuscript; final approval of manuscript submitted. MK Conception, design, analysis and interpretation of data; revising of manuscript; final approval of manuscript submitted. All authors read and approved the final manuscript.

\section{Acknowledgements}

We acknowledge and thank the contributions of all nurses, administrators and physicians at the participating HF Clinics, in addition to members of the steering committee and ICES advisory group.

Clinic name: MD Nurse \& Administrators

Cornwall Heart Failure Clinic: P. De Young and M. Watt

Kitchener-St. Mary's Hospital: S. Smith, D. Lowry, C. Rinne, G. Heckman

Minto Mapleton-Family Health Team: V. LaForge

Credit Valley Hospital: H. Strauss, S. Tierney, J. Cyriac, J. Burtcher, S. Kremer, R. Gandhi, M. Druck, J. Jovanovic, J. Nikhil, K. Nagi, M.Maingi

Burlington Mememorial Hospital: B. Stevens, D. Weber

Hotel Dieu Hospital: J. McCans, P. Staples, W. Earle

Picton-Prince Edward Family Health Team: P. Wattam

University Health Network: H. Ross, L. Belford, D. Delgado, P. Billia, P. Liu, S. Mohammad

Ottawa, H. Haddad, L. Clark, L. Mielniczuk

Trillium A. Gupta, D. Button, D. Harrison, P. Kannampuzha, T. Kalaparambath, M. Kirigin, C. Lazaam, C. LeFeuvre, G. Puley, T. Rebane, B. Sevitt, M. Platinov, L. Singh, R. Watson, S. Tishler, V. fluxar

Orillia Soldier's Memorial Hospital: J. MacFadyen, D. Campbell, S. Crewe, J. Jefferies, D. Bhatt

Georgian Bay Family Health Team: E. Goode, G. Edwards

North York General Hospital: A. Cheng, J. Coldwell, R. James, S. Klein, K. Kwok, B. Lubelsky, P. Myron, R. Rose, M. Strauss, Earl teitelbaum

London Health Sciences Centre: P. Pflugfelder, J. Hoffman

London Health Sciences Centre: M. Arnold, A. Smith

Kitchener New Vision-Family Health Team: A. Horton

Queensway-Carleton Hospital: F. Miller, J. Steele, R. Grewal, T. McKibbin, Lindsey

Ross Memorial: N. Krishnan, C. Follet, D. Kazhila

Mt. Sinai Hospital: S. Mak, A. Schofield, G. Newton, J. Parker, E. Azevedo

Oakville Trafalgar Memorial Hospital: M. Heffernan, J. Orfi, D. McConachie, R.

Mao, V. Chiamvimonvat, M. Feneck

South Lake Regional: J. Symmmes, T. Fair, P. Hacker, L. Blair, R. Chun, J. Allen,

M. Srivamadevan, J. Habot, M. Thangaroopan

Scarborough-General Site: S. Roth, E. King, J Cherry, E. Davies, A. Rosenbloom, G. Vertes, K. Yared

Mt Forest: K. Shelig, D. Horrigan

Owen Sound-Grey Brice Health Sciences Center: A. Becks

Hamilton Health Sciences Center: R. McKelvie and L. Paul

St. Michael's Hospital: A. Al-Hesayen: G. Moe

Toronto East General Hospital: V. Ovchinnikov and S. Garner

Sunnybrook Hospital: R. Myers

Kitchener Center for family medicine: B. Ruby

Steering Committee: H. Abrams, D. Alter, M. DeMelo, F. Wagner, M. Parry, K. Kingsbury, S. Tierney and M. Arnold

Institute for Clinical Evaluative Sciences (ICES) Advisory: J.V. Tu, P. Austin, X. Wang

\section{Funding sources}

Dr. Murray Krahn holds the F. Norman Hughes Chair in Pharmacoeconomics at the Faculty of Pharmacy, University of Toronto. This analysis was funded by funding provided to The Toronto Health Economics and Technology Assessment (THETA) Collaborative by the Ministry of Health and Long-Term Care of Ontario (MOHLTC). The funding organization did not have any role in the design and conduct of the study; collection, management, analysis, and interpretation of the data; and preparation, review, or approval of the manuscript. The opinions, results and conclusions reported in this paper are those of the authors and are independent from the funding sources. No endorsement by the Ontario MOHLTC is intended or should be inferred.

\section{Author details}

Division of Cardiology, Schulich Heart Centre, Sunnybrook Health Sciences Centre, Ontario, Canada. ${ }^{2}$ Toronto Health Economics and Technology Assessment (THETA) Collaborative, Ontario, Canada. ${ }^{3}$ Department of Medicine, University of Toronto, Ontario, Canada. Institute for Work \& Health, Ontario, Canada. ${ }^{5}$ Cardiac Care Network of Ontario, Ontario, Canada. ${ }^{6}$ University Health Network - Toronto General Hospital, Ontario, Canada. ${ }^{7}$ Faculty of Pharmacy, University of Toronto, Ontario, Canada.

Received: 16 October 2011 Accepted: 31 July 2012

Published: 3 August 2012

\section{References}

1. Arnold JM, Liu P, Demers C, Dorian P, Giannetti N, Haddad H, Heckman GA, Howlett JG, Ignaszewski A, Johnstone DE, Jong P, McKelvie RS, Moe GW, Parker JD, Rao V, Ross HJ, Sequeira EJ, Svendsen AM, Teo K, Tsuyuki RT, White M: Canadian Cardiovascular Society consensus conference recommendations on heart failure 2006: diagnosis and management. Can J Cardiol 2006, 22:23-45.

2. Johansen H, Strauss B, Arnold JM, Moe G, Liu P: On the rise: The current and projected future burden of congestive heart failure hospitalization in Canada. Can J Cardiol 2003, 19:430-5.

3. McAlister FA, Stewart S, Ferrua S, McMurray JJ: Multidisciplinary strategies for the management of heart failure patients at high risk for admission: a systematic review of randomized trials. J Am Coll Cardiol 2004, 44:810-9.

4. Komenda P, Levin A: Analysis of cardiovascular disease and kidney outcomes in multidisciplinary chronic kidney disease clinics: complex disease requires complex care models. Curr Opin Nephrol Hypertens 2006 15:61-6.

5. Wright FC, De Vito C, Langer B, Hunter A: Multidisciplinary cancer conferences: a systematic review and development of practice standards. Eur J Cancer 2007, 43:1002-10.

6. Hunt SA, Abraham WT, Chin MH, Feldman AM, Francis GS, Ganiats TG, Jessup M, Konstam MA, Mancini DM, Michl K, Oates JA, Rahko PS, Silver MA, Stevenson LW, Yancy CW: 2009 Focused update incorporated into the ACC/AHA 2005 Guidelines for the Diagnosis and Management of Heart Failure in Adults A Report of the American College of Cardiology Foundation/American Heart Association Task Force on Practice Guidelines Developed in Collaboration With the International Society for Heart and Lung Transplantation. J Am Coll Cardiol 2009, 53:e1-e90.

7. Clark AM, Savard LA, Thompson DR: What is the strength of evidence for heart failure disease-management programs? J Am Coll Cardiol 2009, 54:397-401

8. Medical Advisory Secretariat: Community-Based High Acuity Care for the Specialized Management of Heart Failure: an evidence-based analysis. Ontario Heath Technology Assessment Series: Ontario Heath Technology Assessment Series; 2009:9.

9. Salganik MJ: Variance estimation, design effects, and sample size calculations for respondent-driven sampling. J Urban Health 2006, 83:198-112.

10. Gholizadeh L, Salamonson Y, Worrall-Carter L, DiGiacomo M, Davidson PM: Awareness and causal attributions of risk factors for heart disease among immigrant women living in Australia. J Womens Health (Larchmt) 2009, 18:1385-93.

11. Gustafsson L, Hodge A, Robinson M, McKenna K, Bower K: Information provision to clients with stroke and their carers: self-reported practices of occupational therapists. Aust Occup Ther J 2010, 57:190-6.

12. Rankin J, Bhopal R: Understanding of heart disease and diabetes in a South Asian community: cross-sectional study testing the 'snowball' sample method. Public Health 2001, 115:253-60.

13. Tu K, Gong Y, Maaten S: Physician Care of Patients with Congestive Heart Failure I. In Primary Care in Ontario: ICES Atlas. Edited by Upshur R, KleinGeltink JE, Leong A, Maaten S, Schultz SE, Wang L. Toronto: Institute for Clinical Evaluative Sciences; 2006. 2012.

14. Riegel B, Lee CS, Sochalski J: Developing an instrument to measure heart failure disease management program intensity and complexity. Circ Cardiovasc Qual Outcomes 2010, 3:324-30.

15. Kelly G: The Psychology of Personal Constructs; 1955

16. Rugg G, McGeorge P: The sorting techniques: a tutorial paper on card sorts, picture sorts and item sorts. Expert Systems 1997, 14:80-93.

17. Lindenfeld J, Albert NM, Boehmer JP, Collins SP, Ezekowitz JA, Givertz MM, Katz SD, Klapholz M, Moser DK, Rogers JG, Starling RC, Stevenson WG, Tang 
WH, Teerlink JR, Walsh MN: HFSA 2010 Comprehensive Heart Failure Practice Guideline. J Card Fail 2010, 16:e1-194.

18. Albert NM, Fonarow GC, Yancy CW, Curtis AB, Stough WG, Gheorghiade M Heywood JT, McBride M, Mehra MR, O'Connor CM, Reynolds D, Walsh MN: Outpatient cardiology practices with advanced practice nurses and physician assistants provide similar delivery of recommended therapies (findings from IMPROVE HF). Am J Cardiol 2010, 105:1773-9.

19. Gohler A, Januzzi JL, Worrell SS, Osterziel KJ, Gazelle GS, Dietz R, Siebert U: A systematic meta-analysis of the efficacy and heterogeneity of disease management programs in congestive heart failure. J Card Fail 2006, 12:554-67.

20. Gonseth J, Guallar-Castillon P, Banegas JR, Rodriguez-Artalejo F: The effectiveness of disease management programmes in reducing hospital re-admission in older patients with heart failure: a systematic review and meta-analysis of published reports. Eur Heart J 2004, 25:1570-95.

21. Phillips CO, Singa RM, Rubin HR, Jaarsma T: Complexity of program and clinical outcomes of heart failure disease management incorporating specialist nurse-led heart failure clinics. A meta-regression analysis. Eur $\lrcorner$ Heart Fail 2005, 7:333-41.

22. Roccaforte R, Demers C, Baldassarre F, Teo KK, Yusuf S: Effectiveness of comprehensive disease management programmes in improving clinical outcomes in heart failure patients. A meta-analysis. Eur J Heart Fail 2005, 7:1133-44.

23. Randomised trial of telephone intervention in chronic heart failure: Randomised trial of telephone intervention in chronic heart failure. DIAL trial. BMJ 2005, 331:425-30

24. Del Sindaco D, Pulignano G, Minardi G, Apostoli A, Guerrieri L, Rotoloni M, Petri G, Fabrizi L, Caroselli A, Venusti R, Chiantera A, Giulivi A, Giovannini E, Leggio F: Two-year outcome of a prospective, controlled study of a disease management programme for elderly patients with heart failure. [see comment]. Journal of Cardiovascular Medicine 2007, 8(5):324-9.

25. Doughty RN, Wright SP, Pearl A, Walsh HJ, Muncaster S, Whalley GA, Gamble G, Sharpe N: Randomized, controlled trial of integrated heart failure management: The Auckland Heart Failure Management Study. Eur Heart J 2002, 23:139-46.

26. Dunagan WC, Littenberg B, Ewald GA, Jones CA, Emery VB, Waterman BM, Silverman DC, Rogers JG: Randomized trial of a nurse-administered, telephone-based disease management program for patients with heart failure. J Card Fail 2005, 11:358-65.

27. Holland R, Battersby J, Harvey I, Lenaghan E, Smith J, Hay L: Systematic review of multidisciplinary interventions in heart failure. Heart 2005, 91:899-906.

28. Inglis SC, Clark RA, McAlister FA, Ball J, Lewinter C, Cullington D, Stewart S, Cleland JG: Structured telephone support or telemonitoring programmes for patients with chronic heart failure. Cochrane Database Syst Rev; 2010. CD007228.

29. Mejhert M, Kahan T, Persson H, Edner M: Limited long term effects of a management programme for heart failure. Heart 2004, 90:1010-5.

30. Stromberg A, Martensson J, Fridlund B, Levin LA, Karlsson JE, Dahlstrom U: Nurse-led heart failure clinics improve survival and self-care behaviour in patients with heart failure: results from a prospective, randomised trial. Eur Heart J 2003, 24:1014-23.

31. Jaarsma T, van der Wal MH, Lesman-Leegte I, Luttik ML, Hogenhuis J, Veeger NJ, Sanderman R, Hoes AW, van Gilst WH, Lok DJ, Dunselman PH, Tijssen JG, Hillege HL, van Veldhuisen DJ: Effect of moderate or intensive disease management program on outcome in patients with heart failure: Coordinating Study Evaluating Outcomes of Advising and Counseling in Heart Failure (COACH). Arch Intern Med 2008, 168:316-24.

32. Driscoll A, Worrall-Carter L, Hare DL, Davidson PM, Riegel B, Tonkin A, Stewart S: Evidence-based chronic heart failure management programs: reality or myth? Qual Saf Health Care 2009, 18:450-5.

33. Gustafsoon F, Nielsen P, Hildebrandt P, Ulriksen H, Villadsen H, Andersen B: Prevalence and characteristics of heart failure clinics in Denmark design of the Danish heart failure clinics network. Eur J Heart Fail 2012, 7(2005):283-284

doi:10.1186/1472-6963-12-236

Cite this article as: Wijeysundera et al.: Specialized multi-disciplinary heart failure clinics in Ontario, Canada: an environmental scan. BMC Health Services Research 2012 12:236.

\section{Submit your next manuscript to BioMed Central and take full advantage of:}

- Convenient online submission

- Thorough peer review

- No space constraints or color figure charges

- Immediate publication on acceptance

- Inclusion in PubMed, CAS, Scopus and Google Scholar

- Research which is freely available for redistribution 